\title{
Space-time modeling of soil moisture: Stochastic rainfall forcing with heterogeneous vegetation
}

\author{
I. Rodríguez-Iturbe, ${ }^{1}$ V. Isham, ${ }^{2}$ D. R. Cox, ${ }^{3}$ S. Manfreda, ${ }^{1,4}$ and A. Porporato ${ }^{5}$ \\ Received 9 August 2005; revised 12 October 2005; accepted 19 October 2005; published 25 March 2006.
}

[1] The present paper complements that of Isham et al. (2005), who introduced a space-time soil moisture model driven by stochastic space-time rainfall forcing with homogeneous vegetation and in the absence of topographical landscape effects. However, the spatial variability of vegetation may significantly modify the soil moisture dynamics with important implications for hydrological modeling. In the present paper, vegetation heterogeneity is incorporated through a two dimensional Poisson process representing the coexistence of two functionally different types of plants (e.g., trees and grasses). The space-time statistical structure of relative soil moisture is characterized through its covariance function which depends on soil, vegetation, and rainfall patterns. The statistical properties of the soil moisture process averaged in space and time are also investigated. These properties are especially important for any modeling that aggregates soil moisture characteristics over a range of spatial and temporal scales. It is found that particularly at small scales, vegetation heterogeneity has a significant impact on the averaged process as compared with the uniform vegetation case. Also, averaging in space considerably smoothes the soil moisture process, but in contrast, averaging in time up to 1 week leads to little change in the variance of the averaged process.

Citation: Rodríguez-Iturbe, I., V. Isham, D. R. Cox, S. Manfreda, and A. Porporato (2006), Space-time modeling of soil moisture: Stochastic rainfall forcing with heterogeneous vegetation, Water Resour. Res., 42, W06D05, doi:10.1029/2005WR004497.

\section{Introduction}

[2] Soil moisture dynamics result from the combined action of climate, soil and vegetation. Their space-time characterization may provide significant insight into the description of hydrological, meteorological and ecological processes acting over a variety of scales [e.g., Western and Blöschl, 1999; Rodríguez-Iturbe, 2000; Porporato and Rodríguez-Iturbe, 2002]. Moreover, knowledge of the temporal and spatial variability of soil moisture is critical for understanding and predicting processes such as the partitioning of received energy at the land surface, vegetation water stress in a heterogeneous landscape, soil carbon and nitrogen cycles, etc. The spatial distribution of soil moisture has also been recognized as important in conditioning the hydrological response during extreme events [Manfreda et al., 2005].

[3] Soil moisture dynamics at a point have been extensively analyzed through the use of stochastic models [e.g., Milly, 1993; Rodríguez-Iturbe et al., 1999; Laio et al., 2001;

\footnotetext{
${ }^{1}$ Department of Civil and Environmental Engineering, Princeton University, Princeton, New Jersey, USA.

${ }^{2}$ Department of Statistical Science, University College London, London, UK.

${ }^{3}$ Nuffield College, University of Oxford, Oxford, UK.

${ }^{4}$ Now at Dipartimento di Ingegneria e Fisica dell'Ambiente, Università degli Studi della Basilicata, Potenza, Italy.

${ }^{5}$ Department of Civil and Environmental Engineering, Duke University, Durham, North Carolina, USA.
}

Copyright 2006 by the American Geophysical Union. 0043-1397/06/2005WR004497
Fernandez-Illescas et al., 2001; Porporato et al., 2004]. The stochastic approach leads to a quantitative description of vegetation water stress combining mean intensity, duration and frequency of periods with water deficit [Porporato et al., 2001; Caylor et al., 2005], and to the probabilistic analysis of the nitrogen cycle [Porporato et al., 2003; D'Odorico et al., 2003].

[4] Regarding spatial dynamics, much effort has focused on the analysis of field measurements through remote sensing or ground-based techniques and the importance of physical characteristics such as soil texture, vegetation and topographic patterns for soil moisture variability has been pointed out [Western et al., 2002; Wilson et al., 2004]. Soil moisture retrievals obtained from field campaigns (e.g., Washita '92, SGP '97, '99, SMEX '02 and '04) have allowed the extension of the soil moisture analysis to larger domains, but confined to the near surface [e.g., RodriguezIturbe et al., 1995; Kim and Barros, 2002]. Using the data from SGP '97, Mohanty and Skaggs [2001] analyzed the temporal variability of soil moisture through the time stability concept [Vachaud et al., 1985]. They observed time stable features in more permeable soils and a larger spatiotemporal variability in a flat topography with the coexistence of wheat and grass cover. Yoo and Kim [2004] adopted empirical orthogonal function analysis of the data set identifying topography as the main controlling factor, while vegetation and soil texture have a secondary effect that becomes more significant during the drying periods.

[5] The impact of spatial heterogeneity of soil properties on the soil moisture budget has been extensively investi- 
gated using the scaling theory of soil hydraulic properties [Miller and Miller, 1956]. Milly and Eagleson [1987] analyze the effects of soil heterogeneity on the long-term water balance using the model proposed by Eagleson [1978]. They define the effective soil parameters for an equivalent homogeneous soil to capture effects of the spatial heterogeneity on the soil water budget. Kim et al. [1997] show that equivalent parameters depend not only on the spatial characteristics of soils but also on the climate. Recent results of Zhu and Mohanty [2003] suggest how to derive effective soil parameters.

[6] Other studies [e.g., Albertson and Montaldo, 2003] focus on the description of root zone soil moisture through the development of a conservation equation for the variance of soil moisture within a grid cell with uniform soil and vegetation. This approach identified the effects of processes such as infiltration, evapotranspiration and horizontal redistribution in increasing or decreasing the variance of soil moisture. In particular, it was found that some processes are either solely dissipative or solely productive, while transpiration and infiltration may increase or reduce variance depending on the soil moisture state. Using the same framework, Teuling and Troch [2005] analyze the soil moisture patterns at three sites in different parts of the world emphasizing the significant role of vegetation and soil texture on the soil moisture patterns, vegetation being the dominant control during the growing season.

[7] Despite the previous efforts, many aspects of soil moisture spatial dynamics are not yet clear owing especially to the multitude of sources of variability. In the present work the spatial and temporal dynamics of soil moisture are studied assuming homogeneous soil and negligible topographic effects. The analysis centers on a stochastic model that accounts for the spatial and temporal variability of rainfall and incorporates effects of spatial heterogeneity of vegetation. The approach, although a very simplified representation of an enormously complex problem, yields an analytical framework for the description of the spacetime dynamics of relative soil moisture that clearly indicates the role of vegetation and rainfall parameters under general conditions. This type of framework may be very useful in the attempt to establish general criteria for the description of the soil moisture over a range of spatial and temporal scales.

\section{Space-Time Rainfall Model}

\subsection{Specification of Rainfall Model}

[8] Rainfall events are modelled as a space-time Poisson process of rate $\lambda_{R}$ per unit time per unit area. Each event is characterized as a circular region (or rain cell) of random radius, $W_{R}$, duration, $D$, and intensity, $X$. The model is a special case of that of Cox and Isham [1988] in which we take here the cells to have zero velocity. The rain cells are thus characterized by three random quantities and to keep the number of model parameters small we take the distributions of $W_{R}, D$ and $X$ to be exponential, with means $\mu_{W_{R}}=$ $1 / \rho_{R}, \mu_{D}=1 / \eta$ and $\mu_{X}=1 / \beta$ respectively. All random variables are mutually independent and independent of the process of occurrences. The model is highly idealized but has enough flexibility to represent the main features of the process and is at the same time analytically tractable.
[9] We denote the rainfall intensity at position $\mathbf{u}$ and time $t$ by $Y(\mathbf{u}, t)$, omitting for simplicity the tilde used by Isham et al. [2005]. We also note that here $\lambda_{R}, \rho_{R}$ and $W_{R}$ have been given a subscript $R$ (to denote rain cells) that was not needed in the earlier paper. Then [Isham et al., 2005] the expectation of the rainfall intensity is

$$
E[Y(\mathbf{u}, t)]=\mu_{Y}=\frac{\lambda_{R}^{\prime}}{\eta \beta}
$$

where $\lambda_{R}^{\prime}=2 \pi \lambda_{R} / \rho_{R}^{2}$ is the rate of rain cells that cover an arbitrary space-time point. The variance of the intensity is

$$
\sigma_{Y}^{2}=\frac{2 \lambda_{R}^{\prime}}{\eta \beta^{2}}
$$

The space-time correlation structure of the rainfall intensity process is given by

$$
\operatorname{corr}[Y(\mathbf{0}, t), Y(\mathbf{l}, t+h)]=\lambda_{R}^{\prime \prime}(l) e^{-\eta h} / \lambda_{R}^{\prime}
$$

where $|\mathbf{l}|=l, \lambda^{\prime \prime}{ }_{R}(l)=\lambda_{R} E\left[W_{R}^{2} C\left(l / W_{R}\right)\right]$, and

$$
C(u)= \begin{cases}2 \cos ^{-1}(u / 2)-u\left(1-u^{2} / 4\right)^{\frac{1}{2}} & 0 \leq u \leq 2 \\ 0 & 2 \leq u\end{cases}
$$

is the area of overlap of two unit discs, with centers a distance $u$ apart. For many purposes, $C(u)$ is adequately approximated [see Isham et al., 2005] by

$$
C_{k}(u)= \begin{cases}\pi(1-u / 2) & 0 \leq u \leq 2 \\ 0 & 2 \leq u\end{cases}
$$

leading to the simplified form

$$
\lambda_{R}^{\prime \prime}(l)=\lambda_{R}^{\prime}\left(1+\frac{\rho_{R} l}{4}\right) e^{-\rho_{R} l / 2}
$$

and, in this case, the correlation is approximately

$$
\operatorname{corr}[Y(\mathbf{0}, t), Y(\mathbf{l}, t+h)]=\left(1+\frac{\rho_{R} l}{4}\right) e^{-\rho_{R} l / 2} e^{-\eta h} .
$$

[10] A more detailed description of the derivation of (1) to (2) is given by Isham et al. [2005, section 2] along with a fuller description of the rainfall model.

\subsection{Rainfall Process Aggregated in Time}

[11] Rainfall is a continuous intermittent process generally recorded as cumulative amounts over equispaced time intervals, such as hours or days. Therefore temporally aggregated rainfall is of much interest for hydrologists and meteorologists [Rodríguez-Iturbe et al., 1998].

[12] The formulation of a continuous time random process representing the instantaneous rate of rainfall described in the previous section leads to the derivation of all the most relevant properties at daily or other timescale accumulation.

[13] The aggregated process may be defined as

$$
Y^{T}(\mathbf{u}, t)=\int_{k T}^{(k+1) T} Y(\mathbf{u}, t) d t
$$



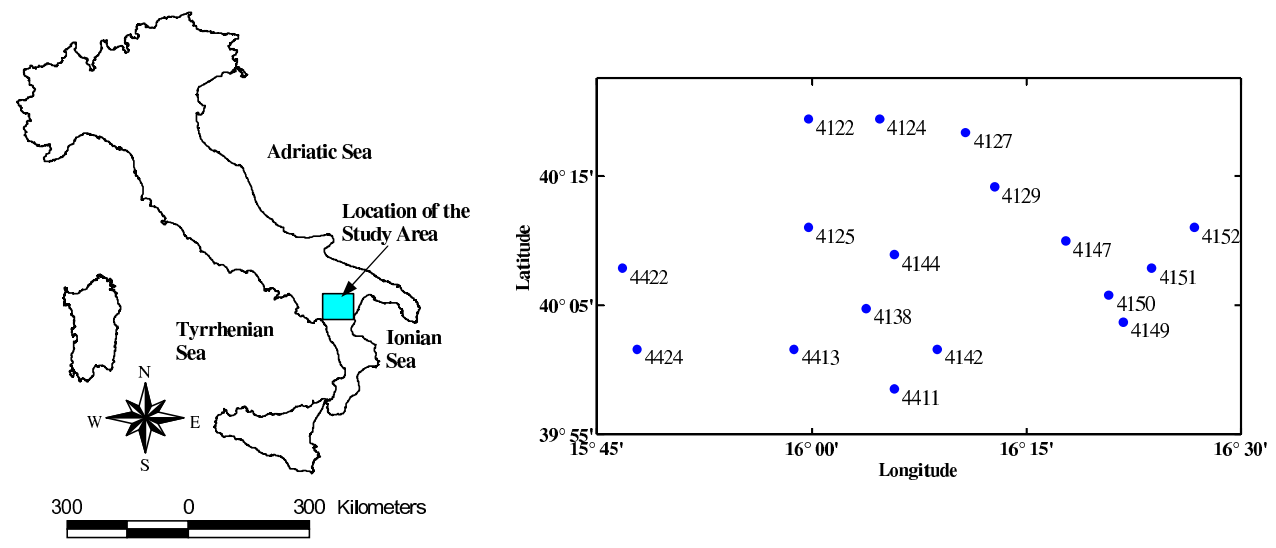

Figure 1. Rainfall network within the Basilicata study area used to estimate the parameters of the rainfall model.

where $[k T,(k+1) T]$ is the time window of integration with $k$ representing the time lag from a time origin and $T$ the aggregation interval.

[14] The mean of the temporally aggregated rainfall is then simply $E\left[Y^{T}(\mathbf{u}, t)\right]=\mu_{Y^{T}}=\mu_{Y} T$, while the variance is

$$
\sigma_{Y^{T}}^{2}=2 \sigma_{Y}^{2}\left(e^{-\eta T}+\eta T-1\right) / \eta^{2} .
$$

[15] The covariance is defined for lags equivalent to multiples $k$ of the interval of aggregation, $T$, and may be derived from the expression

$\operatorname{cov}\left[Y^{T}(\mathbf{0}, 0), Y^{T}(\mathbf{l}, k)\right]=\int_{k T}^{(k+1) T} \int_{0}^{T} \operatorname{cov}\left[Y\left(\mathbf{0}, t_{1}\right), Y\left(\mathbf{l}, t_{2}\right)\right] d t_{1} d t_{2}$

[16] After integration of equation (10) the space-time correlation structure of the temporally aggregated rainfall is obtained in approximate form as

$$
\operatorname{corr}\left(Y^{T}(\mathbf{0}, 0), Y^{T}(\mathbf{l}, k)\right)=\gamma_{S}(l) \gamma_{T}(k)
$$

where

$$
\gamma_{S}(l)=\lambda_{R}^{\prime \prime}(l) / \lambda_{R}^{\prime}
$$

and

$$
\begin{aligned}
\gamma_{T}(k) & =e^{-\eta k T}\left(e^{-\eta T}-2+e^{\eta T}\right) /\left\{2\left(e^{-\eta T}+\eta T-1\right)\right\} \\
k & =1,2, \cdots
\end{aligned}
$$

The special cases of purely spatial and purely temporal correlation are recovered from the individual factors.

[17] These expressions for the mean, variance and correlation function of the aggregated rainfall process will now be used to fit the rainfall model to data from a region in southern Italy.

\subsection{Estimation of the Rainfall Model Parameters}

[18] The study area is part of the Basilicata Region in southern Italy and overlaps two important river basins, the Agri and the Sinni. Daily records of 17 rain gauges covering an area of about $3000 \mathrm{~km}^{2}$ (see Figure 1) were used for
10 years of records without interruption, from 1975 to 1984 in all stations.

[19] To account for the strong seasonality effects typical of Mediterranean climates, we subdivided each record into two periods of six months each: April-September and October-March. The stations are listed in Table 1, where we report also their annual means as well as their seasonal means and standard deviations. The spatial structure of the network is shown in Figure 1.

[20] Estimation of the rainfall model parameters is done as follows.

[21] 1 . The mean rain cell radius $\left(\rho_{R}^{-1}\right)$ is estimated by fitting the spatial correlation function of the temporally aggregated process, $\gamma_{S}(l)$, to the spatial correlation of the data. Figure 2 shows the results for the study area where the parameter $\rho_{R}$ is estimated as $0.080\left(\mathrm{~km}^{-1}\right)$ for the fall/winter period and as $0.060\left(\mathrm{~km}^{-1}\right)$ for the spring/summer period. In the case reported in Figure $2 \mathrm{a}$, we used a multiplying factor equal to 0.7 to remove the discontinuity at zero lag (sometimes called a nugget effect).

[22] 2. The mean storm duration $\left(\eta^{-1}\right)$ is estimated by fitting the temporal correlation function of the aggregated rainfall process, $\gamma_{T}(k)$, to the means of the daily data. Seasonal fluctuations have been removed by subtracting the monthly means from the record. Figure 3 compares the estimated and fitted correlation functions for the spring/ summer season. The temporal correlations in the fall/winter period are negligibly different and have not been shown. The estimate of $\eta$ is 4.05 (day $^{-1}$ ) for the spring/summer season and is $3.75\left(\right.$ day $\left.^{-1}\right)$ for the fall/winter period.

[23] 3 . The mean cell arrival rate $\left(\lambda_{R}\right)$ and the mean cell depth $\left(\beta^{-1}\right)$ are estimated using the equations for the mean and variance of the aggregated rainfall process. Thus one obtains the system,

$$
\begin{gathered}
\lambda_{R}=\frac{2 \rho_{R}^{2}\left(e^{-\eta T}+\eta T-1\right) \mu_{Y^{T}}^{2}}{\pi T^{2} \eta \sigma_{Y^{T}}^{2}}, \\
\beta=\frac{4\left(e^{-\eta T}+\eta T-1\right) \mu_{Y^{T}}}{T \eta^{2} \sigma_{Y^{T}}^{2}},
\end{gathered}
$$

where the mean, $\mu_{Y^{T}}$, and variance, $\sigma_{Y^{T}}^{2}$, of rainfall are estimated directly from the data averaging across all the rain stations. 
Table 1. Rainfall Stations of the Selected Study Area Located in the Basilicata Region, Southern Italy

\begin{tabular}{|c|c|c|c|c|c|c|}
\hline \multirow[b]{2}{*}{ Code } & \multirow[b]{2}{*}{ Name } & \multirow[b]{2}{*}{ Annual Rainfall, mm/yr } & \multicolumn{2}{|c|}{ Spring/Summer } & \multicolumn{2}{|c|}{ Fall/Winter } \\
\hline & & & Mean, mm/d & $\sigma, \mathrm{mm} / \mathrm{d}$ & Mean, mm/d & $\sigma, \mathrm{mm} / \mathrm{d}$ \\
\hline 4138 & Agromonte & 1276 & 2.21 & 5.85 & 4.73 & 10.83 \\
\hline 4124 & Armento & 743 & 1.50 & 5.19 & 2.69 & 7.81 \\
\hline 4144 & Carbone & 1025 & 1.85 & 5.34 & 3.63 & 9.21 \\
\hline 4413 & Castelluccio Inferiore & 1373 & 2.02 & 6.17 & 5.03 & 12.22 \\
\hline 4125 & Castelsaraceno & 1421 & 1.93 & 5.85 & 4.56 & 11.69 \\
\hline 4149 & Cersosimo & 936 & 1.91 & 6.78 & 3.61 & 10.63 \\
\hline 4422 & Lagonegro & 1729 & 3.23 & 9.05 & 7.25 & 16.62 \\
\hline 4127 & Missanello & 816 & 1.72 & 5.59 & 2.52 & 7.08 \\
\hline 4122 & Montemurro & 862 & 1.63 & 4.88 & 2.83 & 7.21 \\
\hline 4150 & Noepoli & 766 & 0.73 & 3.68 & 2.10 & 8.11 \\
\hline 4129 & Roccanova & 671 & 1.26 & 4.19 & 2.27 & 6.55 \\
\hline 4151 & S. Giorgio Lucano & 814 & 1.53 & 6.03 & 3.07 & 13.23 \\
\hline 4142 & S. Severino Lucano & 1410 & 2.28 & 6.40 & 5.30 & 12.68 \\
\hline 4147 & Senise & 632 & 1.13 & 3.63 & 2.24 & 6.44 \\
\hline 4424 & Trecchina & 1927 & 2.77 & 7.80 & 6.68 & 15.23 \\
\hline 4152 & Valsinni & 739 & 1.23 & 5.14 & 2.55 & 10.26 \\
\hline 4411 & Viggianello & 1069 & 1.87 & 5.57 & 4.29 & 9.95 \\
\hline
\end{tabular}

[24] Equations (14) and (15) applied to the study area provided the following estimates for the parameters: $\lambda_{R}=$ $1.66 \times 10^{-4}\left(\mathrm{~d}^{-1} \mathrm{~km}^{-2}\right)$ and $\beta=0.0394(\mathrm{~d} / \mathrm{mm})$, so that $\mu_{X}=25.4 \mathrm{~mm} / \mathrm{d}$, during the spring/summer period; $\lambda_{R}=$ $3.86 \times 10^{-4}\left(\mathrm{~d}^{-1} \mathrm{~km}^{-2}\right)$ and $\beta=0.0263(\mathrm{~d} / \mathrm{mm})$, so that, $\mu_{X}=38.0 \mathrm{~mm} / \mathrm{d}$, for the fall $/$ winter period.

[25] Table 2 summarizes the estimated parameter values of the rainfall model for the two periods under consideration. Values are significantly different between the two periods reflecting the different precipitation patterns observed in the region.

[26] From the model one may estimate the fraction of time that an arbitrary site has rain. Thus for the spring/ summer period, we have [Cox and Isham, 1988] $P_{\text {wet }}=1-$ $\exp \left(-\lambda_{R}^{\prime} / \eta\right)=0.069$. On average a cell is estimated to last 0.25 days, so that it rains once in every $0.25 / 0.069=3.6$ days. By comparison, in the data $18 \%$ of days have some rain, which implies that it is raining at any given site of the

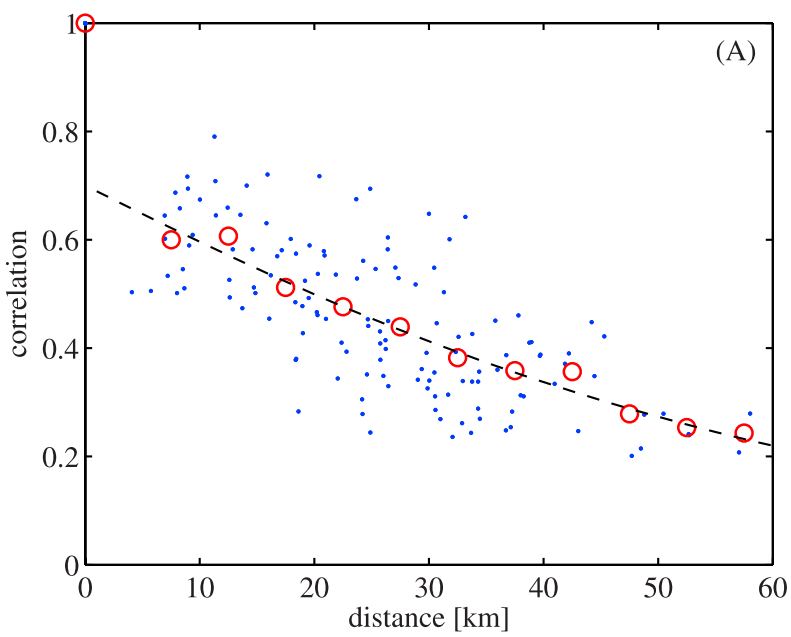

region $18 \times 0.25=4.5 \%$ of the time. Similarly for the fall/ winter period, from the model an arbitrary site of the region is estimated to have rain for $9.6 \%$ of the time. A cell is estimated to last 0.27 days and in the data $24 \%$ of days are wet yielding a corresponding value of $24 \times 0.27=6.5 \%$. Thus the model reproduces fairly well the fraction of time with rain observed in the data.

\section{Soil Moisture Dynamics}

[27] The soil moisture dynamics are described by a simplified water balance equation where the rainfall represents an additive term affected by a factor $(1-\phi)$ that accounts for canopy interception. The soil losses are modelled as a linear function of the relative saturation of soil that represents a reasonable approximation for arid and semiarid regions where saturation and well watered conditions are relatively rare and evapotranspiration is well

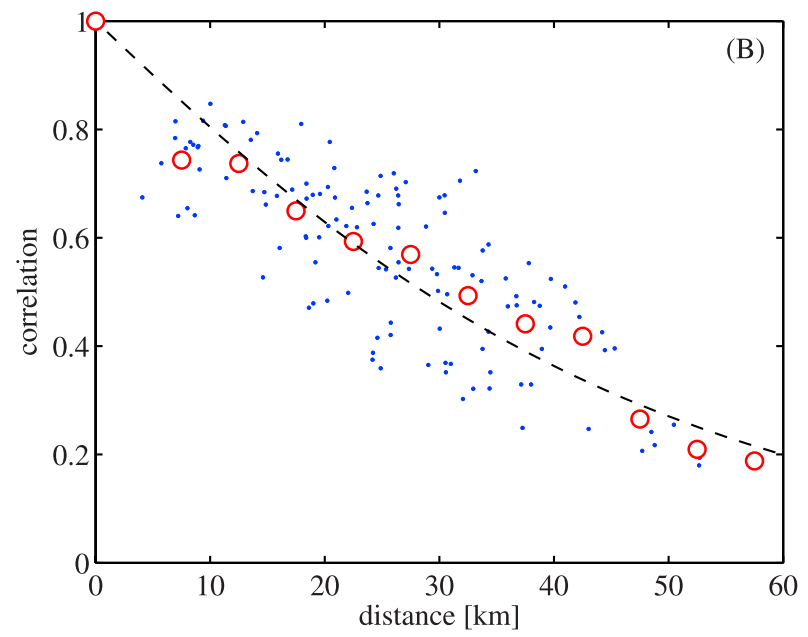

Figure 2. Correlation functions in space of daily precipitation during the periods (a) April-September and (b) October-March in the Basilicata Region. The points describe the correlation between stations grouped by distance, the open circles represent the mean values of the estimated correlation between stations grouped by distance, and the dashed lines are the fitted theoretical spatial correlation given by equation (12) with parameters $\rho_{R}=0.060 \mathrm{~km}^{-1}$ and $\rho_{R}=0.080 \mathrm{~km}^{-1}$, respectively. 


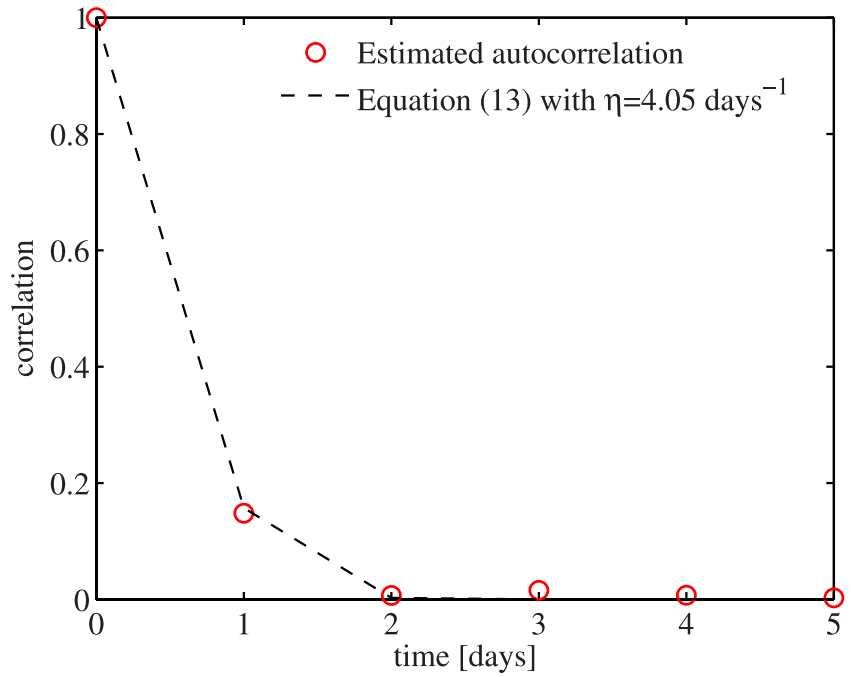

Figure 3. Correlation function in time of the daily precipitation during the period April-September in the Basilicata Region. The circles represent the mean values of the estimated autocorrelation at different stations, and the dashed line is the fitted theoretical autocorrelation given by equation (13) with parameter $\eta=4.05 \mathrm{day}^{-1}$.

approximated by a linear dependence on soil moisture content [Laio et al., 2001; Porporato et al., 2004]. The equation may be written as

$$
n Z_{r} \frac{d S(t)}{d t}=(1-\phi) Y(t)-V S(t)
$$

where $S(t)$ (dimensionless) is the relative soil moisture at time $t ; n$ is the soil porosity; $Z_{r}[\mathrm{~L}]$ is the depth of the root zone; $V[\mathrm{~L} / \mathrm{T}]$ is the soil water loss coefficient; $(1-\phi)$ (dimensionless) is the net rainfall coefficient. The parameter $V$ accounts for evapotranspiration, leakage, and runoff losses; it depends on vegetation and soil characteristics. The factor $(1-\phi)$ is mainly controlled by the plant species and condition of vegetation. Typical values range between 0.6 and 0.9 [e.g., Lull, 1964].

[28] The previous equation can be standardized and rewritten in spatially explicit form for an arbitrary point $A$ as

$$
\frac{d S_{A}(t)}{d t}=-a_{A} S_{A}(t)+b_{A} Y_{A}(t)
$$

where $a_{A}=\left[V /\left(n Z_{r}\right)\right]_{A}$ is the normalized soil water loss at $A$ and $b_{A}=\left[(1-\phi) /\left(n Z_{r}\right)\right]_{A}$ is the normalized net rainfall coefficient that describes the effects of the vegetation interception, assumed to be a constant fraction of the rainfall intensity. Such parameters synthetically account for the effects of vegetation characteristics on soil water balance. The solution of (17) gives the dynamics of the soil moisture process as a function of the rainfall input. Thus, in equilibrium, one obtains

$$
S_{A}(t)=\int_{0}^{\infty} e^{-a_{A} \omega} b_{A} Y_{A}(t-\omega) d \omega
$$

[29] Isham et al. [2005] discuss in detail the impact of the lack of an upper bound in the relative soil moisture dynamics as well as the form of interception used in equation (17).

[30] The expected value of the relative soil moisture given the vegetation at a point is

$$
E\left[S_{A}(t)\right]=\frac{b_{A}}{a_{A}} \mu_{Y}
$$

[31] The covariance of the soil moisture at two points ( $A$ and $B$ ) separated in space by a distance $l$ and in time by an interval $h$ has the form

$$
\begin{aligned}
\operatorname{cov}\left[S_{A}(t), S_{B}(t+h)\right]= & \int_{0}^{\infty} \int_{0}^{\infty} b_{A} b_{B} e^{-a_{A} u-a_{B} v} \\
& \cdot \operatorname{cov}\left[Y_{A}(t-u), Y_{B}(t+h-v)\right] d u d v .
\end{aligned}
$$

[32] Thus, conditionally on a given vegetation cover at the two points, the covariance is obtained from equation (20) as

$$
\begin{aligned}
c_{A B}(h)= & \operatorname{cov}\left[S_{A}(t), S_{B}(t+h)\right] \\
= & \frac{\lambda_{R}^{\prime \prime}(l) \sigma_{Y}^{2} b_{A} b_{B}}{\lambda_{R}^{\prime}} \\
& \times\left(\frac{2 \eta e^{-a_{B} h}}{\left(a_{A}+a_{B}\right)\left(\eta^{2}-a_{B}^{2}\right)}+\frac{e^{-\eta h}}{\left(a_{B}-\eta\right)\left(a_{A}+\eta\right)}\right)
\end{aligned}
$$

where it is important that the site $(B)$, characterized by the parameters $\left(a_{B}, b_{B}\right)$, corresponds to the later time, and that the vegetation cover controls the value of the pairs $\left(a_{A}, b_{A}\right)$ and $\left(a_{B}, b_{B}\right)$.

[33] For vegetation assumed uniform in space, $a_{A}=a_{B}=$ $a$ and $b_{A}=b_{B}=b$, the space-time covariance of relative soil moisture is then

$$
\operatorname{cov}\left[S_{A}(t), S_{B}(t+h)\right]=\frac{\lambda_{R}^{\prime \prime}(l) \sigma_{Y}^{2} b^{2}}{\lambda_{R}^{\prime} a} \frac{\left(\eta e^{-a h}-a e^{-\eta h}\right)}{\left(\eta^{2}-a^{2}\right)}
$$

and has been studied in detail by Isham et al. [2005].

\section{Heterogeneous Vegetation}

\subsection{Specification Vegetation Model}

[34] In ecology, there has been increasing interest in the study of spatial patterns of vegetation and the underlying processes [e.g., Turner, 1989; Levin, 1992]. These patterns result from complex interaction between physical and biological forces and interact with hydrological processes [e.g., Rodriguez-Iturbe and Porporato, 2004]. They have been extensively studied in savanna ecosystems [e.g., Smith and Goodman, 1987; Skarpe, 1991; Jeltsch et al., 1999]. Of

Table 2. Rainfall Model Parameters Estimated Over the Basilicata Region

\begin{tabular}{lll}
\hline \multicolumn{1}{c}{ Parameters } & Spring/Summer Period & Fall/Winter Period \\
\hline$\rho_{R}, \mathrm{~km}^{-1}$ & 0.060 & 0.080 \\
$\eta, \mathrm{day}^{-1}$ & 4.05 & 3.75 \\
$\beta, \mathrm{d} / \mathrm{mm}^{-2} \mathrm{~d}^{-1}$ & 0.0394 & 0.0263 \\
$\lambda_{R}, \mathrm{~km}^{-1}$ & $1.66 \times 10^{-4}$ & $3.86 \times 10^{-4}$ \\
$\lambda_{R}^{\prime}, \mathrm{day}^{-1}$ & 0.289 & 0.379 \\
\hline
\end{tabular}



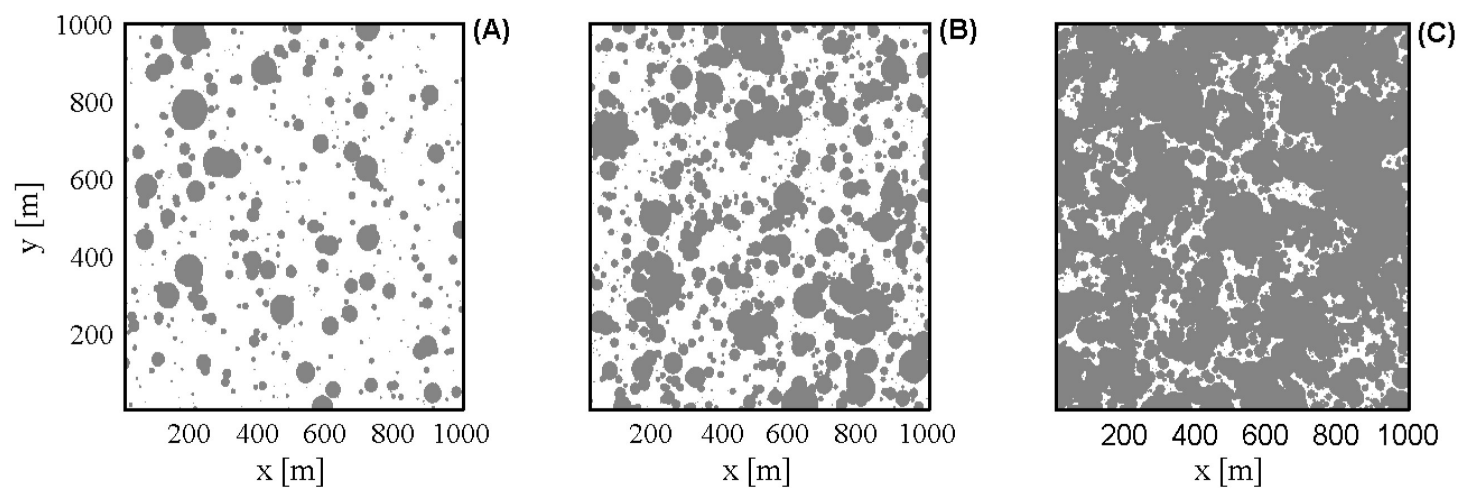

Figure 4. Examples of the vegetation patterns obtained from the model with $\rho_{T}=1 / 8 \mathrm{~m}^{-1}$ and $\lambda_{T}$ equal to 500,1500 , and $5000 \mathrm{~km}^{-2}$ moving from the left to the right; gray represents trees, and white represents the grasses. The tree cover fraction is (a) $18 \%$, (b) $45 \%$, and (c) $87 \%$.

interest here, Caylor et al. [2003] recently examined the spatial arrangement of canopy-dominant trees at 10 sites along the Kalahari Transect in southern Africa showing they are predominantly randomly located.

[35] Here we consider the presence of two functionally different vegetation types (e.g., grasses and trees) whose different physiological characteristics will affect the values of the parameters $a$ and $b$ in the soil moisture equation. The spatial structure of vegetation is represented by assuming a matrix of grass with trees located according to a Poisson process in space with rate $\lambda_{T}$. The trees are assumed to have circular crowns with radii, $W_{T}$, exponentially distributed with parameter $\rho_{T}$. Figure 4 gives some examples of realizations from the above model.

[36] To remove the conditioning on vegetation types at $A$ and $B$ in the covariance function of soil moisture, the probability of occurrence of the four possible combinations of land cover for the two points $(A, B)$ is needed. Once these probabilities are obtained we may then proceed to use the already derived expression for the covariance given the cover at $A$ and $B$ (equation (21)) weighted by the joint probabilities of the vegetation cover. We use the subscript $c$ for the points covered by tree and $u$ for the points uncovered. Thus the parameters $a_{A}$ and $b_{A}$ will take values $a_{c}$ or $a_{u}$, and $b_{c}$ or $b_{u}$ according as $A$ is or is not under the canopy; similarly for the parameters attached to point $B$.

\subsection{Joint Probabilities of Vegetation Cover}

[37] With the vegetation described by the above Poisson model the expressions for the probabilities of the four different possible combinations of vegetation cover at two points $A$ and $B$ separated by a distance $l$ in space are as follows. Denote the event that $A$ is not covered by the canopy by $A_{u}$, with events $A_{c}, B_{u}$ and $B_{c}$ defined similarly. The number of trees covering $A$ has Poisson distribution of mean $\lambda_{T} \mathrm{E}\left(\pi W_{T}^{2}\right)$, so that

$$
P\left(A_{u}\right)=\exp \left\{-\lambda_{T} E\left(\pi W_{T}^{2}\right)\right\}=\exp \left(-2 \pi \lambda_{T} / \rho_{T}^{2}\right) .
$$

Consequently the probability that a point is covered by a tree is

$$
P\left(A_{c}\right)=1-P\left(A_{u}\right)=1-\exp \left(-2 \pi \lambda_{T} / \rho_{T}^{2}\right) .
$$

[38] Similarly the number of trees covering both $A$ and $B$, a distance $l$ apart, has a Poisson distribution with mean $\lambda_{T} E\left\{W_{T}^{2} C\left(l / W_{T}\right)\right\}$. We can now calculate the probabilities of such events as $A_{u} \cap B_{c}$, that $A$ is uncovered and $B$ covered. For this we write

$$
K_{T}=W_{T}^{2}\left\{\pi-C\left(l / W_{T}\right)\right\},
$$

so that $E\left(K_{T}\right)$ is the expected crown area reduced by the expected area of overlap between two tree crowns $l$ apart. The joint probability of having point $A$ not covered and $B$, at distance $l$, covered (or vice versa) is thus

$$
\begin{aligned}
P\left(A_{u} \cap B_{c}\right)= & P\left(A_{c} \cap B_{u}\right)=\exp \left\{-\lambda_{T} E\left(\pi W_{T}^{2}\right)\right\} \\
& \cdot\left(1-\exp \left\{-\lambda_{T} E\left(K_{T}\right)\right\}\right) .
\end{aligned}
$$

[39] In the same way, the probability that two points, at distance $l$ from each other, are both uncovered is

$$
P\left(A_{u} \cap B_{u}\right)=\exp \left\{-\lambda_{T} E\left(\pi W_{T}^{2}+K_{T}\right)\right\} .
$$

Finally, the probability that both points are under cover can be found by subtraction.

[40] The expectation of the tree crown area reduced by the overlapping area may be derived using the simplified expression (5). In this case, when $W_{T}$ is exponentially distributed with parameter $\rho_{T}$,

$$
E\left(K_{T}\right)=2 \pi \rho_{T}^{-2}\left[1-\left(1+\rho_{T} l / 4\right) e^{-\rho_{T} l / 4}\right]
$$

leading to simplified expressions for the probabilities such as $P\left(A_{u} \cap B_{u}\right)$ that have been used in the numerical work later in the paper.

\subsection{Statistical Moments of Relative Soil Moisture}

[41] The expected value of the relative soil moisture in the heterogeneous case may be obtained as the sum of the means of tree covered soil and grass covered (i.e., tree uncovered) soil weighted by their relative probabilities. That is,

$$
E(S)=\mu_{S}=\mu_{Y}\left\{b_{c} / a_{c}+\left(b_{u} / a_{u}-b_{c} / a_{c}\right) \zeta\right\},
$$

where $\zeta=\exp \left(-2 \pi \lambda_{T} / \rho_{T}^{2}\right)$. 

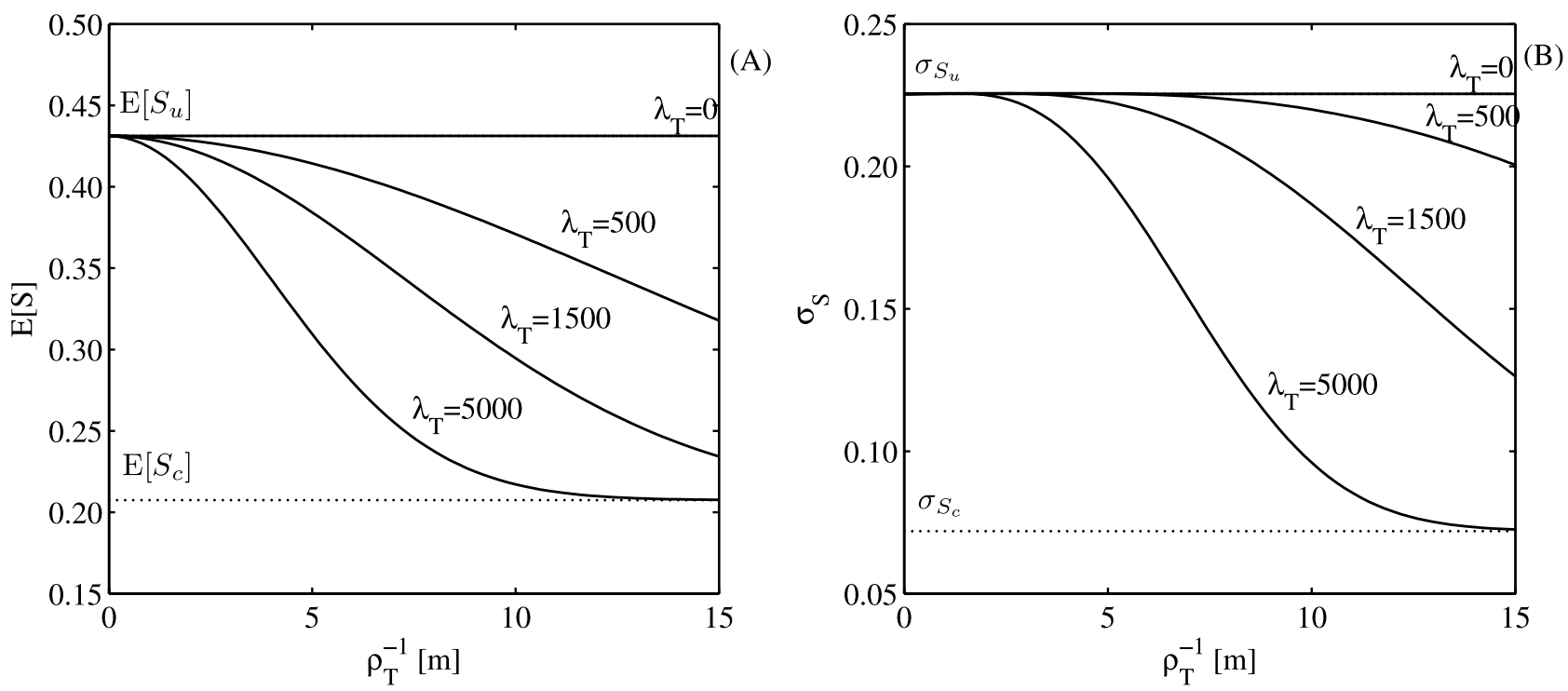

Figure 5. Dependence of the (a) mean and (b) standard deviation of the relative soil moisture on the parameters $\lambda_{T}\left[\mathrm{~km}^{-2}\right]$ and $\rho_{T}\left[\mathrm{~m}^{-1}\right]$ of vegetation. Parameters of the rainfall process are $\lambda_{R}=1.66 \times 10^{-4}$ day $^{-1} \mathrm{~km}^{-2}, \eta=\mu_{D}{ }^{-1}=4.05$ day $^{-1}, \beta=\mu_{X}{ }^{-1}=0.0394 \mathrm{~d} / \mathrm{mm}$, and $\rho_{R}=\mu_{W_{R}}^{-1}=0.060 \mathrm{~km}^{-1}$. Parameters related to the vegetation are $V_{T}=7 \mathrm{~mm} / \mathrm{d}, \phi_{T}=0.2,\left[n Z_{r}\right]_{T}=400 \mathrm{~mm}, V_{G}=4 \mathrm{~mm} / \mathrm{d}, \phi_{G}=0.05$, and $\left[n Z_{r}\right]_{G}=100 \mathrm{~mm}$.

[42] When the entire landscape is covered by trees or by grass, the mean assumes the expression for the uniform case. An example of mean soil moisture as a function of the parameters $\lambda_{T}$ and $\rho_{T}$ is given in Figure 5a. The parameters of the rainfall process used in this example correspond to those of the April-September period for the Basilicata region.

[43] The covariance of the soil moisture for heterogeneous vegetation can be derived from the expression of the conditional covariance function (equation (21)). In general, the covariance between two random variables $Y$ and $Z$ can be obtained from their conditional covariance given $X$ as

$$
\operatorname{cov}(Y, Z)=E_{X}\{\operatorname{cov}(Y, Z \mid X)\}+\operatorname{cov}\left\{E_{X}(Y \mid X), E_{X}(Z \mid X)\right\}
$$

Here we take $X$ as representing cover so that the conditioning on $X$ gives four distinct possibilities of which two, corresponding to one site covered and the other not, are under our assumptions equal by symmetry. Because the relevant probabilities for different cover combinations have been obtained in Section 4.2 and furthermore the covariance conditional on coverage at the two points has also been derived, one can easily obtain the unconditional covariance from equation (29). The analytical solution thus obtained is lengthy and not illuminating and will not be given here. Numerical values are straightforward to calculate from equations (29) and (21).

[44] The variance of the relative soil moisture in a heterogeneous landscape is obtained similarly, but the expressions are simpler since $A$ and $B$ are coincident and therefore only two possibilities for cover must be considered. Thus

$$
\sigma_{S}^{2}=\sigma_{Y}^{2}\left(\frac{b_{u}^{2} \zeta}{a_{u}\left(\eta+a_{u}\right)}+\frac{b_{c}^{2}(1-\zeta)}{a_{c}\left(\eta+a_{c}\right)}\right)+\mu_{Y}^{2}\left(\frac{b_{u}}{a_{u}}-\frac{b_{c}}{a_{c}}\right)^{2} \zeta(1-\zeta) .
$$

[45] Formulas for uniform grass or uniform tree cover follow as special cases. The first term in equation (30) represents the sum of the variances weighted by the probabilities of the two vegetation types; the second term depends on the squared difference between the ratios $b / a$ of the two vegetations coexisting over the landscape. Figure $5 b$ shows the standard deviation of relative soil moisture as a function of the vegetation parameters.

[46] Both mean and standard deviation of relative soil moisture (Figures $5 \mathrm{a}$ and $5 \mathrm{~b}$ ) are represented as functions of the mean radius of tree crowns. As expected, both functions are highly sensitive to $\rho_{T}$ for high values of $\lambda_{T}$.

[47] Examples of the correlation of relative soil moisture are shown in Figure 6 for the set of rainfall parameters estimated for the Basilicata region. The inset shows the same function on a semilog scale to underline the correlation reduction due to the presence of vegetation at small scales that is not clearly distinguishable in the linear plot.

[48] Two regimes can be clearly identified in the correlation structure of the relative soil moisture corresponding to small and large spatial scales. Separation of scales has also been noticed in the analysis of soil moisture data in Illinois (USA) and Russia by Vinnikov et al. [1996, 1999], where the empirical correlation was characterized as the sum of two exponential functions associated with small-scale land surface variability and large-scale atmospheric forcing, respectively. Those authors also observed a slightly higher spatial correlation in the case of a growing forest with a more uniform cover.

\subsection{Relative Soil Moisture Instantaneous in Time and Integrated in Space}

[49] The impact of spatial scale on the description of soil moisture dynamics is an interesting and important topic in hydrology. The extensive use of remote sensing images makes this issue even more critical for a correct interpreta- 


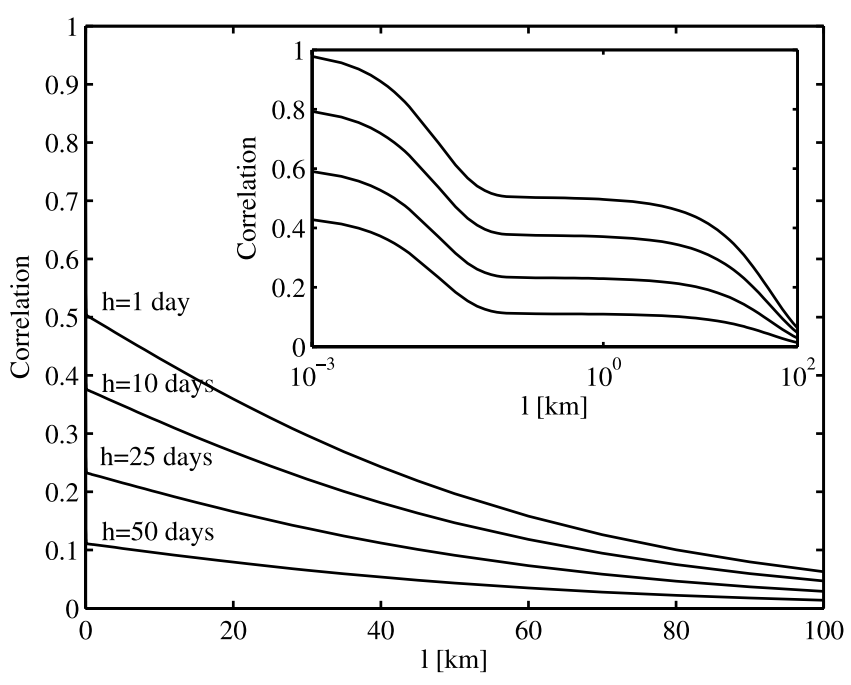

Figure 6. Examples of correlation functions of relative soil moisture for the case of heterogeneous vegetation. Parameters of the rainfall process are $\lambda_{R}=1.66 \times 10^{-4} \mathrm{~d}^{-1}$ $\mathrm{km}^{-2}, \eta=\mu_{D}{ }^{-1}=4.05$ days $^{-1}, \beta=\mu_{X}{ }^{-1}=0.0394 \mathrm{~d} / \mathrm{mm}$, and $\rho_{R}=\mu_{W_{R}}^{-1}=0.060 \mathrm{~km}^{-1}$. Parameters related to the vegetation are $V_{T}=7 \mathrm{~mm} / \mathrm{d}, \phi_{T}=0.2,\left[n Z_{r}\right]_{T}=400 \mathrm{~mm}$, $V_{G}=4 \mathrm{~mm} / \mathrm{d}, \phi_{G}=0.05,\left[n Z_{r}\right]_{G}=100 \mathrm{~mm}, \lambda_{T}=2000 \mathrm{~km}^{-2}$, and $\rho_{T}=0.008 \mathrm{~km}$.

tion of features averaged over different spatial scales, while soil moisture variability within an area may significantly affect the results from hydrological models.

[50] In this Section we analyze the variance of the relative soil moisture process averaged over a square of side $L$. This can be obtained by integrating the correlation function of the soil moisture process in space as [e.g., Vanmarcke, 1983],

$$
\begin{aligned}
\sigma_{S_{L}}^{2}= & \frac{1}{L^{4}} \int_{-L}^{L} \int_{-L}^{L}\left(L-\left|u_{1}\right|\right)\left(L-\left|u_{2}\right|\right) \\
& \cdot \operatorname{cov}\{S(\boldsymbol{w}, t), S(\boldsymbol{w}+\boldsymbol{u}, t)\} d u_{1} d u_{2},
\end{aligned}
$$

where the previous notation has been extended so that $S(\boldsymbol{w}, t)$ is the soil moisture at vector position $\boldsymbol{w}$ at time $t$.

[51] Because of the relative algebraic complexity of the covariance function of relative soil moisture, here we obtain the variance of the integrated process only via numerical integration. For homogeneous vegetation, Isham et al. [2005] found the variance of the integrated process analytically using a Gaussian approximation to the more complex, analytically derived spatial correlation function.

[52] The results described in the following refer to a landscape made up by two functionally different vegetation types, listed in Table 3, and to the climatic conditions of the Basilicata region in southern Italy typical of the period April-September (see Table 2).

[53] Figure 7 compares the standard deviation of the relative soil moisture integrated in space as a function of the integration area for heterogeneous vegetation and for the two alternative homogeneous cases. The vegetation heterogeneity affects the standard deviation of the soil moisture at all scales producing a function that is in between the two bounds represented by the homogeneous cases. For the smaller averaging areas (from $10^{-6}$ to $10^{-1} \mathrm{~km}^{2}$ ), the shape of the function describing the standard deviation of the averaged process differs from that of the two homogeneous cases. This may be related to the spatial characteristics of the correlation function shown in Figure 6. For larger scales the standard deviation of the relative soil moisture has the behavior of a homogeneous case with an averaged cover, because when the averaging area has a characteristic dimension exceeding the correlation length of the vegetation structure, the stochastic effects of the vegetation heterogeneity are largely removed from the process.

[54] Figure 8 shows the ratio between the standard deviation of the spatially averaged relative soil moisture and the standard deviation at a point for different values of $\rho_{R}$ for both the homogeneous and the heterogeneous vegetation cases. The spatial averaging produces a significant reduction of the point standard deviation for areas larger than $50 \mathrm{~m} \times 50 \mathrm{~m}$ in the heterogeneous case while the homogeneous case does not show a reduction of the above ratio at this scale. The reduction becomes dominant for areas above $1 \mathrm{~km}^{2}$ in both cases.

[55] The behavior of the ratio between the variance of the spatially averaged process and the point variance underlines the importance of allowing for heterogeneous vegetation for a correct description of the relative soil moisture, especially at the small scales where the vegetation structure represents a dominant component. In fact, differences in rainfall parameters $\left(\rho_{R}\right.$ and $\left.\lambda_{R}\right)$ have a negligible effect for averaging areas of less than $10^{-1} \mathrm{~km}^{2}$. On the other hand, variability of the averaged process becomes mainly controlled by the parameter $\rho_{R}$ at scales larger than $1 \mathrm{~km}^{2}$. It was also found that averaging over areas of characteristic size larger than $100 / \rho_{R}$ results in a loss of the structure overimposed by the rainfall field.

[56] Figure 9 shows the standard deviation of the spatially averaged process over a square area of $100 \mathrm{~m} \times 100 \mathrm{~m}$ as a function of the tree root depth multiplied by soil porosity, $n Z_{r}$, and for different root depths of the grass. Soil moisture variability increases with the reduction of the storage capacity represented by $n Z_{r}$, shallow roots leading to larger fluctuations in the soil moisture induced by both rainfall inputs and water losses. Furthermore, the soil moisture variability displays a large sensitivity to the presence of shallow-rooted vegetation within the landscape. In fact, changes in the product $\left[n Z_{r}\right]_{G}$ for grasses lead to larger modifications in the standard deviation of the relative soil moisture than those induced by the corresponding product $\left[n Z_{r}\right]_{T}$ for trees.

\subsection{Relative Soil Moisture Averaged in Space and Time}

[57] The dependence of relative soil moisture on the averaging time does not significantly differ from the homo-

Table 3. Parameters Adopted to Characterize the Two Vegetation Types Considered Within the Model

\begin{tabular}{cl}
\hline Vegetation Parameters & Value \\
\hline$\left[n Z_{r}\right]_{T}, \mathrm{~mm}$ & 400 \\
$\phi_{T}$ & 0.20 \\
$V_{T}, \mathrm{~mm} / \mathrm{d}$ & 7.0 \\
$\lambda_{T}, \mathrm{~km}^{-2}$ & 2000 \\
$\rho_{T}^{-1}, \mathrm{~km}$ & 0.0080 \\
{$\left[n Z_{r}\right]_{G}, \mathrm{~mm}$} & 100 \\
$\phi_{G}$ & 0.050 \\
$V_{G}, \mathrm{~mm} / \mathrm{d}$ & 4.0 \\
\hline
\end{tabular}




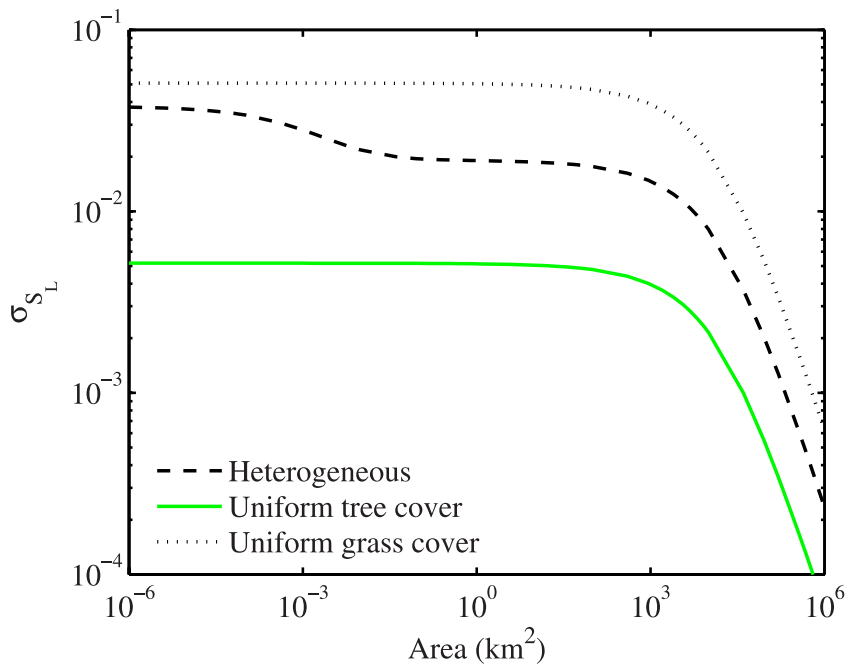

Figure 7. Standard deviation of the spatially integrated soil moisture in the case of heterogeneous vegetation and uniform vegetation. Parameters are as for Figure 6.

geneous case discussed by Isham et al. [2005]. In fact, the time-dependent terms of the covariance function are not modified by the presence of heterogeneous vegetation (whose characteristics are assumed here to be timeindependent).

[58] Figure 10 shows an example of the standard deviation of the averaged process over an area of $1 \mathrm{~km}^{2}$ and for integration windows ranging from 1 day to 30 days. Modest changes occur for temporal scales ranging from 1 day to 3 days and only after 3 days does the standard deviation start to decrease in a more noticeable manner. Although Figure 10 corresponds to a particular set of parameter values this behavior was observed for many other examples. Timescales lower than 1 day are not shown since the loss function used in the model neglects all dynamics occurring at shorter timescales and thus is appropriate only at scales of

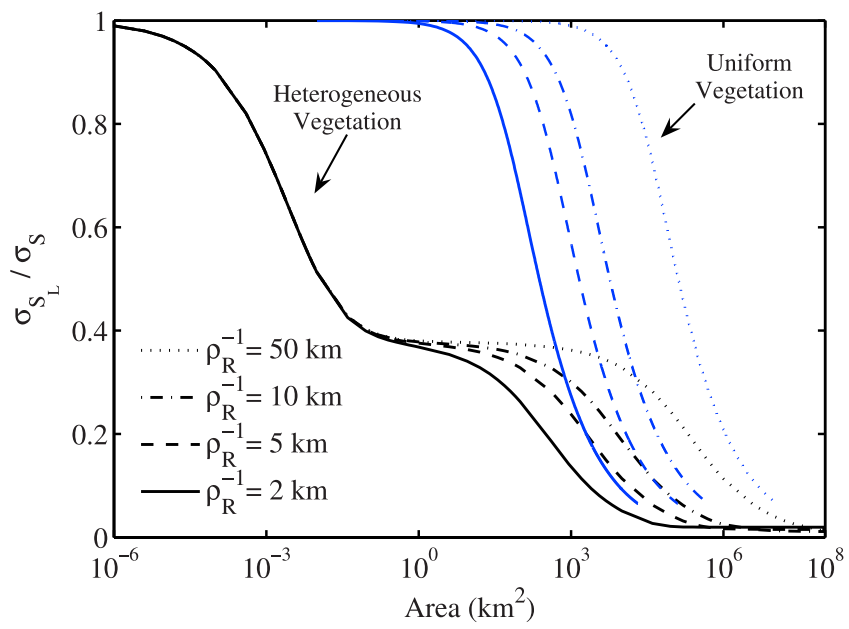

Figure 8. Ratio between the standard deviation of the spatially averaged relative soil moisture and the point standard deviation for different $\rho_{R}$ for both the homogeneous and heterogenous vegetation cases. Remaining parameters are as for Figure 6.

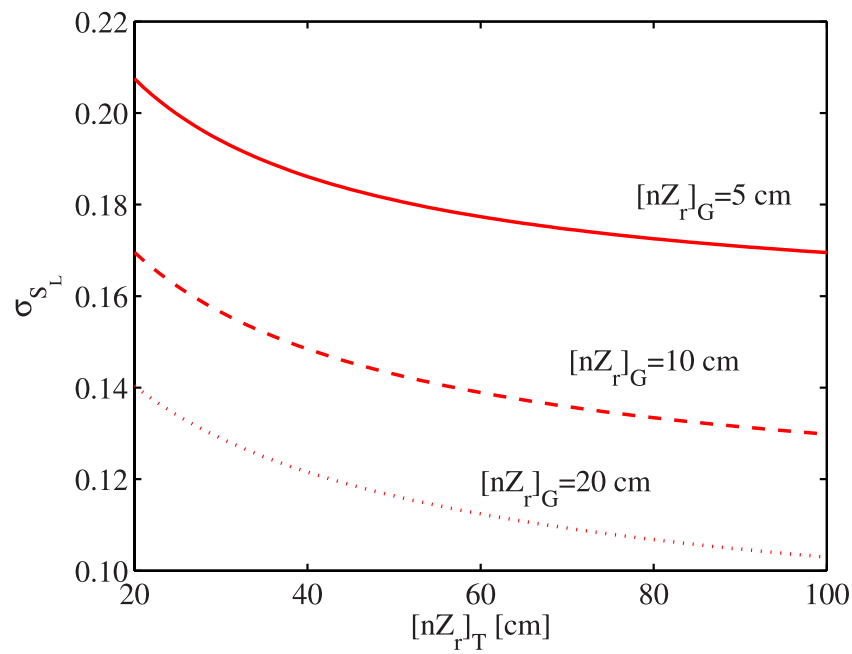

Figure 9. Standard deviation of the soil moisture averaged over a square area of $0.01 \mathrm{~km}^{2}$ as a function of the parameter $\left[n Z_{r}\right]_{T}[\mathrm{~cm}]$ and for three different values of $\left[n Z_{r}\right]_{G}$. The remaining parameters are as for Figure 6 .

a day or more. It should be noted, however, that apart from sudden soil moisture increases due to the pulsing rainfall input (that are properly accounted for in the model), other processes acting at the hourly timescale are not expected to introduce marked temporal variability in soil moisture dynamics and thus to affect its temporal autocorrelation at short time lags.

[59] Figure 11 shows the impact of averaging in both space and time on the standard deviation of the relative soil moisture process. The graph is obtained via numerical integration for averaging areas ranging from $1 \mathrm{~m}^{2}$ to $10^{6} \mathrm{~km}^{2}$ and using time intervals of 1,10 and 30 days. The importance of scales for a correct characterization of the variability of the soil moisture process is clearly detected. Averaging in time induces modifications to the standard deviation that are minor relative to those resulting from averaging in space. With uniform soil texture conditions

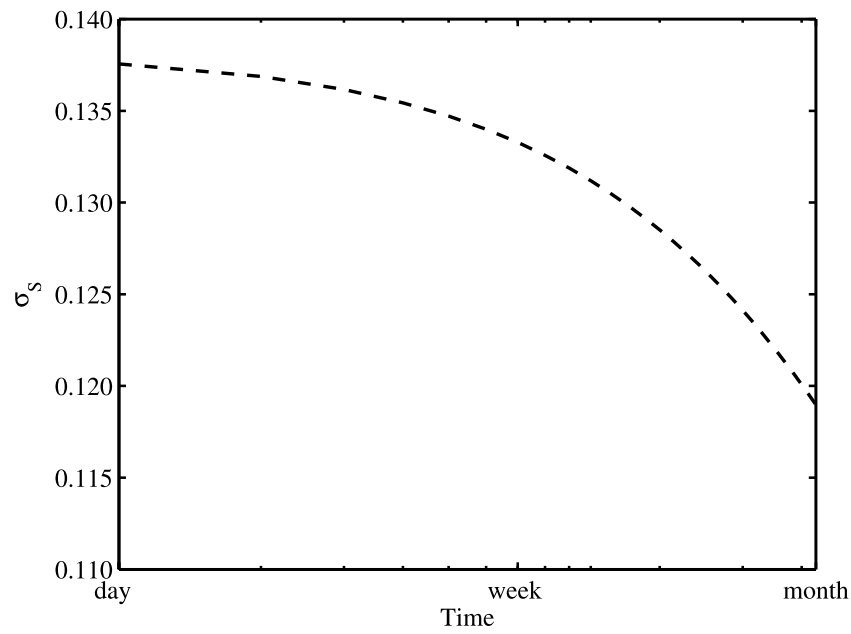

Figure 10. Standard deviation of the relative soil moisture averaged over a square area of $1 \mathrm{~km}^{2}$ and in time over durations ranging from 1 day to 1 month. Parameters are as for Figure 6. 


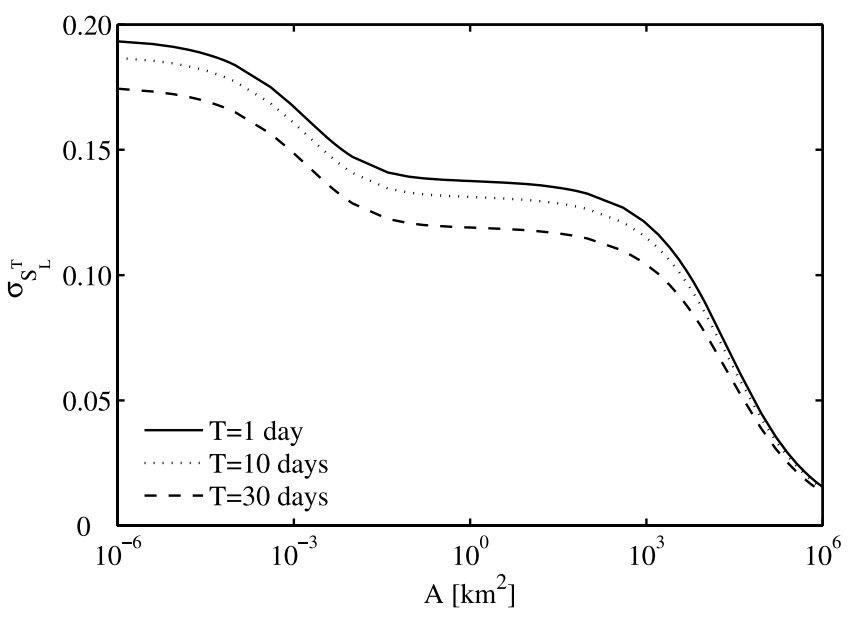

Figure 11. Standard deviation of relative soil moisture for the heterogeneous vegetation case as a function of the size of the averaging area and for different time intervals $T=1$, 10 , and 30 (days). Parameters are as for Figure 6.

the spatial correlation of the relative soil moisture results from two interacting fields, one operating at small spatial scales (vegetation) and the other at larger scales (rainfall). The first sharp reduction of the standard deviation is due to the averaging at small scales that transforms the heterogeneous landscape into averaged homogeneous vegetation while the second sharp reduction is due to the decrease of correlation induced by the rainfall field.

\section{Final Discussion}

[60] The spatial and temporal variability of the soil moisture has been described through an analytical formalism that incorporates effects of rainfall (e.g., spatial structure and temporal dynamics), soil properties (e.g., effective root depth and leakage) and vegetation (e.g., spatial heterogeneity of the landscape, rainfall interception and evapotranspiration) in the covariance function of the process. The covariance reflects the dynamics of the soil moisture resulting from the interactions of processes acting over different spatial scales. The variance of the relative soil moisture is found to be proportional to the rainfall variance and related to the parameters of the two functionally different vegetation types and the structure of the vegetation cover (see equation (30)).

[61] The most important conclusions from this study are now briefly described.

[62] Vegetation composition has a significant impact on the variability of the averaged process, especially at small scales, and becomes progressively less significant with the increase of the averaging area. Particularly interesting is the ratio between the variance of the spatially averaged soil moisture and the point variance (see Figure 8) which is quite different from that found for the homogeneous case. This ratio is considerably smaller than one for averaging areas larger than $50 \mathrm{~m} \times 50 \mathrm{~m}$ showing that the vegetation structure significantly modifies soil moisture variability. Hydrological modeling should therefore account for this in the parametrization of spatially averaged soil moisture and fluxes dependent on this variable.
[63] The standard deviation of the averaged relative soil moisture is particularly sensitive to the averaging spatial scale. Averaging in space considerably smoothes the relative soil moisture process; this effect depends on the vegetation characteristics (small scale) and the structure of the rainfall process (large scale). By contrast, averaging in time up to periods of 1 week does not modify significantly the soil moisture standard deviation. It is important to remark here that this result is likely to be strongly dependent on the assumption that no topographical effects are controlling the soil moisture dynamics. In regions where largescale topography plays a dominant role, time averaging could have a significant effect.

[64] Comparison of the derived analytical results with the space-time correlation structure of soil moisture fields is a research priority of the authors. We are not aware of empirical soil moisture data available in space and time that will enable a strict validation of the theory. For this reason we are proceeding to compare the analytical results with correlation structures derived from soil moisture fields obtained through different types of hydrologic models. The results of such analyses will be reported elsewhere.

[65] The impact of controlling topographical effects on the space-time correlation structure of soil moisture is an important concern of current research. Previous work by Caylor et al. [2005] has highlighted the importance of the interlocked systems of hillslopes and the drainage network in the spatial organization of soil moisture content. The authors are attempting to link a modified version of the scheme presented in this paper to the spatial structure of a river basin where the drainage network acts as a template for the organization of the soil moisture dynamics.

[66] Acknowledgments. I.R.-I. and S.M. gratefully acknowledge the support of NOAA under grant NA17RJ2612 and NSF under the National Center for Earth Surface Dynamics (NCED) (EAR-0120914). A.P. acknowledges the Office of Science, Biological and Environmental Research Program, U.S. Department of Energy, through the Great Plains Regional Center of the National Institute for Global Environmental Change under cooperative agreement DEFC02-03ER63613.

\section{References}

Albertson, J. D., and N. Montaldo (2003), Temporal dynamics of soil moisture variability: 1. Theoretical basis, Water Resour. Res., 39(10), 1274, doi:10.1029/2002WR001616.

Caylor, K. K., H. H. Shugart, P. R. Dowty, and T. M. Smith (2003), Tree spacing along the Kalahari Transect in southern Africa, J. Arid Environ., 54(2), 281-296.

Caylor, K. K., S. Manfreda, and I. Rodríguez-Iturbe (2005), On the coupled geomorphological and ecohydrological organization of river basins, $A d v$. Water Resour., 28(1), 69-86.

Cox, D. R., and V. Isham (1988), A simple spatial-temporal model of rainfall, Proc. R. Soc., Ser. A, 415, 317-328.

D’Odorico, P., F. Laio, A. Porporato, and I. Rodríguez-Iturbe (2003), Hydrologic controls on soil carbon and nitrogen cycles. II A case study, Adv. Water Resour, 26(1), 59-70.

Eagleson, P. S. (1978), Climate, soil, and vegetation: 1. Introduction to water balance dynamics, Water Resour. Res., 14(5), 705-712.

Fernandez-Illescas, C. P., A. Porporato, F. Laio, and I. Rodríguez-Iturbe (2001), The ecohydrological role of soil texture in a water-limited ecosystem, Water Resour. Res., 37, 2863-2872.

Isham, V., D. R. Cox, I. Rodríguez-Iturbe, A. Porporato, and S. Manfreda (2005), Representation of space-time variability of soil moisture, Proc. $R$. Soc., Ser. A, 461(2064), 4035-4055, doi:10.1098/rspa.2005.1568.

Jeltsch, F., K. Moloney, and S. J. Milton (1999), Detecting process from snapshot pattern: Lessons from tree spacing in the southern Kalahari, Oikos, 85, 451-466. 
Kim, G., and A. P. Barros (2002), Space-time characterization of soil moisture from passive microwave remotely sensed imagery and ancillary data, Remote Sens. Environ., 81, 393-403.

Kim, C. P., J. N. M. Stricker, and R. A. Feddes (1997), Impact of soil heterogeneity on the water budget of the unsaturated zone, Water Resour. Res., 33(5), 991-999.

Laio, F., A. Porporato, C. P. Fernandez Illescas, and I. Rodríguez-Iturbe (2001), Plants in water-controlled ecosystems: Active role in hydrologic processes and response to water stress. IV: Discussion of real cases, $A d v$. Water Resour., 24, 745-762.

Levin, S. A. (1992), The problem of pattern and scale in ecology, Ecology, $73,1943-1967$.

Lull, H. W. (1964), Ecological and silvicultural aspects, in Handbook of Applied Hydrology, edit by V. T. Chow, pp. 6-1-6-30, McGraw-Hill, New York.

Manfreda, S., V. Iacobellis, and M. Fiorentino (2005), DREAM: A distributed model for runoff, evapotranspiration, and antecedent soil moisture simulation, Adv. Geosci., 2, 31-39.

Miller, E. E., and R. D. Miller (1956), Physical theory for capillary flow phenomena, J. Appl. Phys., 27, 324-332.

Milly, P. C. D. (1993), An analytical solution of the stochastic storage problem applicable to soil water, Water Resour. Res., 29(11), 37553758.

Milly, P. C. D., and P. S. Eagleson (1987), Effects of spatial variability on annual average water balance, Water Resour. Res., 23(11), 2135-2142.

Mohanty, B. P., and T. H. Skaggs (2001), Spatio-temporal evolution and time-stable characteristics of soil moisture within remote sensing footprints with varying soil, slope, and vegetation, Adv. Water Resour., 24, $1051-1067$.

Porporato, A., and I. Rodríguez-Iturbe (2002), Ecohydrology: A challenging multidisciplinary research perspective, Hydrol. Sci. J., 47(5), $811-822$.

Porporato, A., F. Laio, L. Ridolfi, and I. Rodríguez-Iturbe (2001), Plants in water controlled ecosystems: Active role in hydrological processes and response to water stress. III. Vegetation water stress, Adv. Water Resour., $24,725-744$.

Porporato, A., P. D’Odorico, F. Laio, and I. Rodríguez-Iturbe (2003), Hydrologic controls on soil carbon and nitrogen cycles. I. Modeling scheme, Adv. Water Resour, 26(1), 45-58.

Porporato, A., E. Daly, and I. Rodríguez-Iturbe (2004), Soil water balance and ecosystem response to climate change, Am. Nat., 164(5), 625-633.

Rodríguez-Iturbe, I. (2000), Ecohydrology: A hydrologic perspective of climate-soil-vegetation dynamics, Water Resour. Res., 36(1), 3-9.

Rodríguez-Iturbe, I., and A. Porporato (2004), Ecohydrology of WaterControlled Ecosystems: Soil Moisture and Plant Dynamics, Cambridge Univ. Press, New York.

Rodríguez-Iturbe, I., C. K. Vogel, R. Rigon, D. Entekhabi, F. Castelli, and A. Rinaldo (1995), On the spatial organization of soil moisture fields, Geophys. Res. Lett., 22(20), 2757-2760.

Rodríguez-Iturbe, I., M. Marani, P. D’Odorico, and A. Rinaldo (1998), On the space-time scaling of cumulated rainfall fields, Water Resour. Res., $34(12), 3461-3470$
Rodríguez-Iturbe, I., A. Porporato, L. Ridolfi, V. Isham, and D. R. Cox (1999), Probabilistic modelling of water balance at a point: The role of climate, soil and vegetation, Proc. R. Soc., Ser. A, 455, 3789-3805.

Skarpe, C. (1991), Spatial patterns and dynamics of woody vegetation in an arid savanna, J. Veg. Sci., 2, 565-572.

Smith, T. M., and P. S. Goodman (1987), Successional dynamics in an Acacia nilotica-Euclea divinorum savannah in southern Africa, J. Ecol., 75, 603-610.

Teuling, A. J., and P. A. Troch (2005), Improved understanding of soil moisture variability dynamics, Geophys. Res. Lett., 32, L05404, doi:10.1029/2004GL021935.

Turner, M. G. (1989), Landscape ecology: The effect of pattern on process, Annu. Rev. Ecol. Syst., 20, 171-197.

Vachaud, G., A. Passerat de Silans, P. Balabanis, and M. Vauclin (1985), Temporal persistence of spatially measured soil water probability density function, Soil Sci. Soc. Am. J., 49, 822-828.

Vanmarcke, E. (1983), Random Fields: Analysis and Synthesis, MIT Press, Cambridge, Mass.

Vinnikov, K. Y., A. Robock, N. A. Speranskaya, and C. A. Schlosser (1996), Scales of temporal and spatial variability of midlatitude soil moisture, J. Geophys. Res., 101, 7163-7174.

Vinnikov, K. Y., A. Robock, S. Qiu, and J. K. Entin (1999), Optimal design of surface networks for observation of soil moisture, J. Geophys. Res., $104,4145-4168$

Western, A. W., and G. Blöschl (1999), On the spatial scaling of soil moisture, J. Hydrol., 217, 203-224.

Western, A. W., R. B. Grayson, and G. Blöschl (2002), Scaling of soil moisture: A hydrologic perspective, Annu. Rev. Earth Planet. Sci., 30, $149-180$.

Wilson, D. J., A. W. Western, and R. B. Grayson (2004), Identifying and quantifying sources of variability in temporal and spatial soil moisture observations, Water Resour. Res., 40, W02507, doi:10.1029/ 2003WR002306.

Yoo, C., and S. Kim (2004), EOF analysis of surface soil moisture field variability, Adv. Water Resour., 27(8), 831-842.

Zhu, J., and B. P. Mohanty (2003), Effective hydraulic parameters for steady state vertical flow in heterogeneous soils, Water Resour. Res., 39(8), 1227, doi:10.1029/2002WR001831. UK.

D. R. Cox, Nuffield College, University of Oxford, Oxford OX1 1NF, V. Isham, Department of Statistical Science, University College London, Gower Street, London WC1E 6BT, UK.

S. Manfreda, Dipartimento di Ingegneria e Fisica dell'Ambiente, Università degli Studi della Basilicata, via dell'Ateneo Lucano 10, I-85100 Potenza, Italy. (manfreda@unibas.it)

A. Porporato, Department of Civil and Environmental Engineering, Duke University, Durham, NC 27708, USA.

I. Rodríguez-Iturbe, Department of Civil and Environmental Engineering, Princeton University, Princeton, NJ 08540, USA. 